RESEARCH PAPER RP981

Part of Journal of Research of the National Bureau of Standards, Volume 18, March 1937

\title{
PERFORMANCE CHARACTERISTICS OF A WATER CURRENT METER IN WATER AND IN AIR
}

\author{
By Galen B. Schubauer and Martin A. Mason
}

\begin{abstract}
The effect of density on the performance of a water current meter of the cupwheel type, known as the small Price meter, was investigated by calibrating the meter first in water by towing it in a rating tank, and second in air by placing the meter in a wind tunnel. The change from water to air produced a change in density by a factor of approximately 800 . It was found that the revolutions of the cup wheel during 1 foot of travel of the fluid was a function of the product of velocity by the square root of the density and that Reynolds number and turbulence have no measurable effect. It was concluded that changes of density occurring in field use can cause no appreciable error.
\end{abstract}

CONTENTS

Page

I. Introduction

II. Experimental investigation

III. Dimensional considerations

IV. Results _ _ _

V. Discussion

VI. Conclusion_...

\section{INTRODUCTION}

The development and utilization of the water resources of the country for flood control, irrigation, and power generation require an accurate knowledge of the total quantity of water available in streams, rivers, and watersheds and of the normal flow, the minimum flow in time of drought, and the maximum flow in time of flood of streams and rivers. These data are obtained in part from water velocity measurements made with current meters, and hence the accuracy of the data depends in part on the accuracy of current meters.

Current meters are not absolute instruments and hence must be calibrated. The calibration is generally made in clear, fresh water. However, when used in the field, particularly in time of flood, the water is by no means clear, carrying silt, mud, and debris. Likewise, meters are occasionally used for measurements in salt water. The question immediately arises as to the effect of these departures from the conditions of the calibration on the indications of the meter.

It is known that the mixtures or solutions encountered in the field are more dense than clear, fresh water; but whether or not the effect on the meter is the same as that which would be produced by a homogeneous fluid of the same density cannot be answered simply. Certainly, one effect of silt and mud is to increase the inertia of the 
fluid and to this extent the effect on the indications of the meter will be similar to that of a fluid of greater density. If the water carries particles of considerable magnitude, such as pebbles, or if the mixture is so thick as to be much more viscous than water, other effects are obviously present. Calibrations of a current meter in homogeneous fluids of different density may be expected to give some indication of the behavior of the meter in mixtures or solutions of densities not differing greatly from that of clear, fresh water.

In order to determine the effect of the density on their calibration, two current meters were rated in homogeneous media of different density: (1) in water in the rating tank of the National Bureau of Standards, and (2) in air in the Bureau's 41/2-ft wind tunnel. By this means a density change by a factor of about 800 was secured.

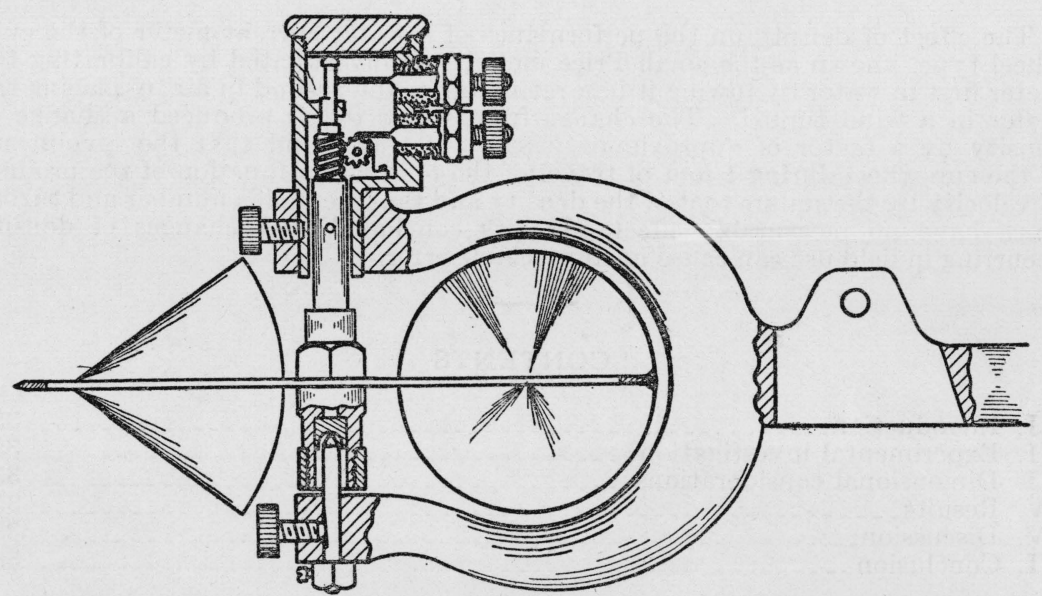

FIGURE 2.-Diagram of small Price water current meter, showing the arrangement of bearings and contact mechanism.

A comparison of the rates of rotation of the meters at the same relative speed of the fluid with respect to the meter was intended to show the effect, if any, of density variation. Since, however, the change from the rating tank to the wind tunnel involved differences in turbulence and Reynolds number, as well as density, the possible effect of these additional variables had to be considered.

The only other work known in which an attempt was made to compare the performance of a water current meter in both water and air is that reported for a propeller type meter in 1901 by Hajós. ${ }^{1}$ Unfortunately, the information given about the tests is too meager to permit an analysis of the results for comparison with the results presented here.

\section{EXPERIMENTAL INVESTIGATION}

The meters used in the study are known as small Price meters (figs. 1 and 2) and are of the cup-wheel type, the wheel revolving about a vertical axis. They depend for their operation on the differential pressure acting on the open and closed ends of the cups. Figure

\footnotetext{
${ }^{1} \mathrm{~S}$. Hajós. Beiträge zur Frage über die Umlaufswerte Woltmann'scher Flügel. Deutsch-Oesterreich Ungarischer Verband für Binnenschifffahrt, new series no. IX (1901).
} 


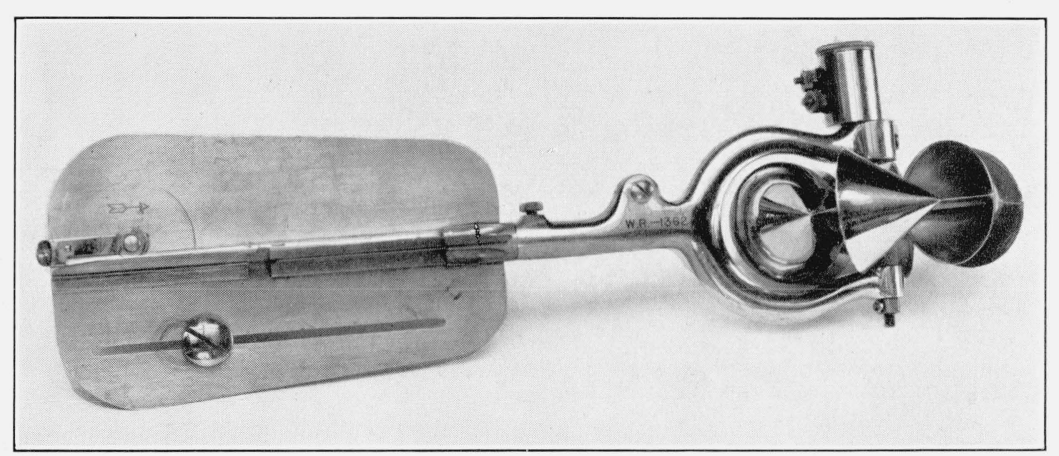

Figure 1. - Small Price water current meter. 
2 shows the constructional details of this type of meter. The calibration of the meter consists in the determination of the relation between the rate of rotation of the cup-wheel and the velocity of the fluid relative to the meter, for the range of velocities expected to be encountered in the field. In the rating tank, the cailibration is made by towing the meter through still water; in the wind tunnel, the calibration is made by drawing air past the stationary meter.

The experimental procedure was arranged as follows.

The two meters were first rated in water, then in air, and again in water to determine whether changes in the meter had occurred during the high-speed running in air. The meters were mounted singly below the towing car 24 inches below the surface of the water on a rod suspension. The same suspension equipment was used in the wind tunnel, the upper end of the rod being attached to the top of the wind tunnel 27 inches above the meter. Complete tests were made of two meters to avoid possible errors resulting from irregularities in any one instrument.

\section{DIMENSIONAL CONSIDERATIONS}

The characteristics of current meters of either the propeller or the cup-wheel type are usually expressed in terms of the "apparent pitch", defined as the actual distance, in feet, traveled by the fluid while the propeller or cup wheel makes one turn. The reciprocal of this quantity, termed "reciprocal of apparent pitch" or simply "revolutions per foot", is more commonly used in connection with the cup-wheel type, for purposes of precise calibration, and for this reason will be used here. If $D$ is the diameter of the cup wheel, in feet, it follows that revolutions per foot $\times \pi D$ is a dimensionless ratio expressing the ratio of the peripheral distance traveled by the cups during rotation for a given period of time to the distance traveled by the fluid in the same time.

If, from the several quantities which may affect the rate of rotation of the cup wheel, we choose only those of importance in the present treatment, we then have:

$U$, velocity of the fluid

$\rho$, density of the fluid

$\mu$, viscosity of the fluid

$\sqrt{\overline{u^{2}}}$, turbulence expressed in terms of the root-mean-square value of the turbulence velocity fluctuations

$D$, some dimension of the instrument, such as the diameter of the cup wheel

$T$, opposing torque arising from bearing and contact friction when no load is imposed by the drag of the cup wheel

$\Delta T$, change in opposing torque resulting from change in bearing friction attributable to the drag of the cup wheel

It is found by dimensional considerations that revolutions per foot $\times \pi D$ may be expressed in terms of dimensionless ratios of the foregoing quantities by the following functional relation:

$$
\text { Revolutions per foot } \times \pi D=F\left(\frac{T+\Delta T}{\rho U^{2} D^{3}}, \frac{U D \rho}{\mu}, \frac{\sqrt{\overline{u^{2}}}}{U}\right),
$$

where $F$ is an unknown function, the nature of which may be determined by experiment. The quantity $U D \rho / \mu$ is the Reynolds number, and $\sqrt{\overline{u^{2}} / U}$ is the intensity of the turbulence. 
Since the drag load on the bearings is proportional to $\rho U^{2} D^{2}$, the change in frictional torque $\Delta T$ may be assumed to be proportional to $\rho U^{2} D^{3}$. If this assumption is correct $\Delta T / \rho U^{2} D^{3}=$ constant.

The assumption is correct provided the geometrical form of the bearings and the coefficient of friction do not depend on the magnitude of the drag. We may, therefore, replace the first member on the right of relation 1 by $\frac{T}{\rho U^{2} D^{3}}+A$, where $\mathrm{A}$ is simply a constant. We may then rewrite the functional relation more simply as

$$
\text { Revolutions per foot }=\frac{1}{\pi D} F_{1}\left(\frac{T}{\rho U^{2} D^{3}}+A, \frac{U D \rho}{\mu}, \frac{\sqrt{\overline{u^{2}}}}{U}\right),
$$

where $F_{1}$ is a new function.

Dimensional considerations show only the combinations of variables upon which the quantity, revolutions per foot, depends. The relative importance of the three members on the right-hand side of relation 2 and the nature of the function can best be found by experiment.

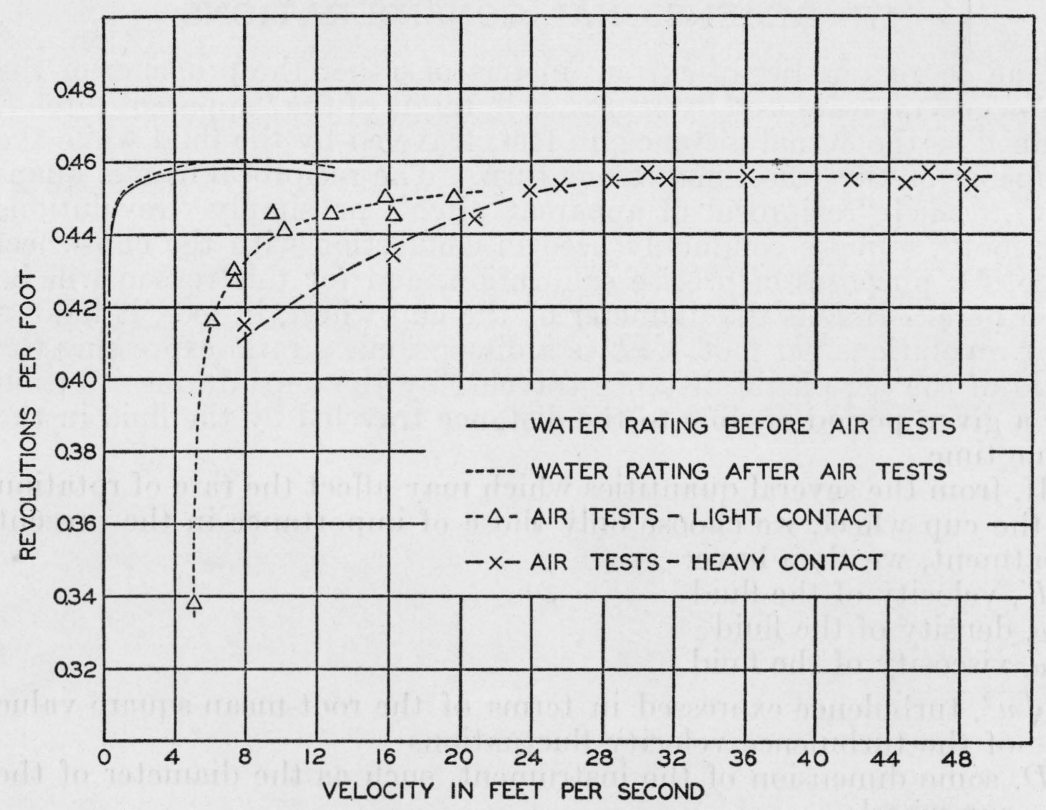

FIGURE 3.-Relation between revolutions per foot and velocity of water and air. Meter WR-1495.

\section{RESULTS}

The results are given in figures 3 to 8 , inclusive. The figures are given in pairs showing similar diagrams for each meter, the pairs consisting of 3 and $4 ; 5$ and $6 ; 7$ and 8 . Figures 3 and 4 each show two sets of curves giving the relation between revolutions per foot and water velocity in one case and air velocity in the other. The curves for the two media differ greatly, especially at the lower velocities. In order to account for the difference between the curves, it is necessary to consider the relative effects of the three members on the right of relation 2 , 


$$
\frac{T}{\rho U^{2} D^{3}}+A, \frac{U D \rho}{\mu}, \frac{\sqrt{\overline{u^{2}}}}{U}
$$

the first two of which differ in the two media because of the change in $\rho$ and $\mu$, and the third because of the change in flow conditions. It is assumed that $T$, which depends on the condition of the bearings and the tension of the contact brush, remains the same in the two media. For a particular value of the velocity the change from water to air raised $T / \rho U^{2} D^{3}$ by a factor of approximately 800 and lowered $U D \rho / \mu$ by a factor of about 13 . The amount of turbulence in the water of the rating tank was unknown, although it was probably smaller than

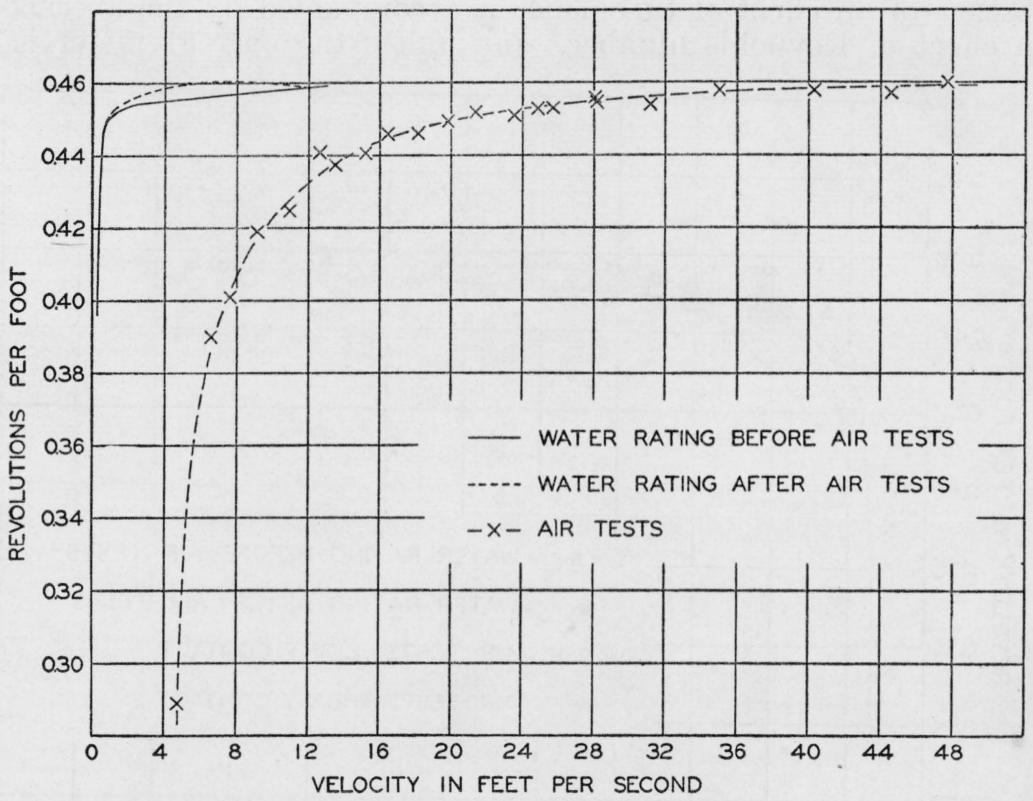

FIGURE 4.-Relation between revolutions per foot and velocity of water and air. Meter WR-1496.

that in the wind tunnel, since the water in the rating tank was allowed to become sensibly still between each observation run.

The intensity of turbulence in the wind tunnel under ordinary conditions was 0.85 percent $\left(100 \sqrt{\overline{u^{2}}} / U=0.85\right)$. Fortunately, it was possible in the wind tunnel to vary $\sqrt{\overline{u^{2}}} / U$ independently of $T / \rho U^{2} D^{3}$ and $U D \rho / \mu$. This was accomplished by placing completely across the upstream section of the tunnel a square-mesh screen made of round wooden rods $5 / 8$ inch in diameter, spaced $3 \frac{114}{4}$ inches apart. ${ }^{2}$ At the current-meter location, which was 7.6 feet downstream from the screen, the intensity of the turbulence was 2.7 percent. After a thorough investigation of the velocity distribution at the current-meter loca-

\footnotetext{
- For a more detailed account of this method of producing turbulence and the nature of the turbulence itself see: Measurements of Intensity and Scale of Wind-Tunnel Turbulence and their Relation to the Critical Reynolds Number of Spheres. Ntl. Advisory Comm. Aero. Tech Rept. 581 (1937).
} 
tion, both meters were run at this higher turbulence. By comparing runs made at the higher turbulence with those given in figures 3 and 4 , which correspond to the lower turbulence, it was found for both meters that the rates were the same for 2.7-percent turbulence as for 0.85 percent; or if any differences did exist, they were too small to be detected above the experimental scatter. In contrast to this result, the rate of a Robinson cup anemometer of the old standard type with four smooth hemispherical cups was found to be increased by 5 percent at the higher turbulence. It was concluded, therefore, that the term $\sqrt{\overline{u^{2}}} / U$ could be dropped from relation 2 as being without effect on the rating of cup-wheel current meters of the small Price type.

It has been found generally in aerodynamic investigations that an absence of an effect of turbulence is accompanied by the absence of an effect of Reynolds number, ${ }^{3}$ and unless the current meter is an

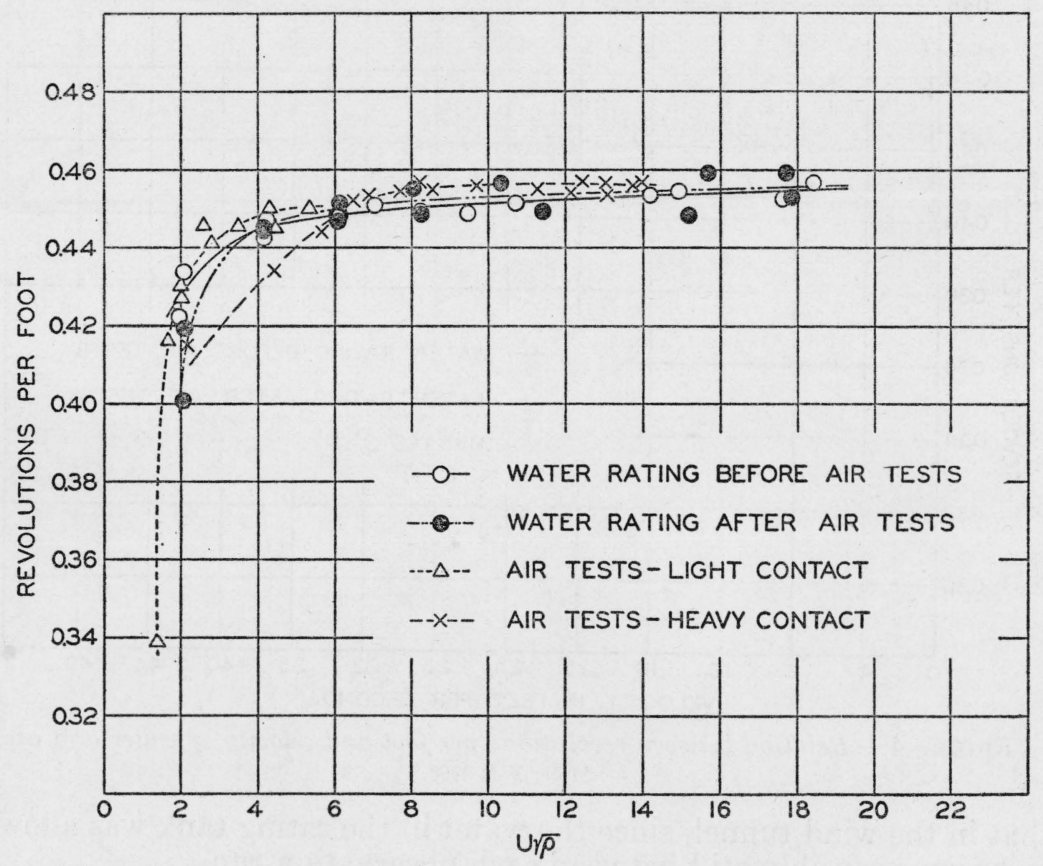

FiguRe 5.-Relation between revolutions per foot and $U \sqrt{\rho}$.

$U=$ velocity of water or air in feet per second.

$\rho=$ Density of water or air, in pounds per cubic foot. Meter WR-1495.

exception, the same result which permits the dropping of $\sqrt{\overline{u^{2}}} / U$ suggests also the omission of $\mathrm{UD} \rho / \mu$ in relation 2 . The tests with two different amounts of turbulence lead, therefore, to the supposition that relation 2 might be simplified to

$$
\text { Revolutions per foot }=\frac{1}{\pi D} F_{2}\left(\frac{T}{\rho U^{2} D^{3}}+A\right) \text {, }
$$

or with $T$ and $D$ constant and $\rho U^{2}$ as the only variable quantity

Revolutions per foot $=F_{3}(U \sqrt{\rho})$,

\footnotetext{
${ }^{3}$ Hugh L. Dryden, Turbulence companion of Reynolds number. J. Aero. Sci. 1, no. 2, 67 (April 1934).
} 
where the new function $F_{3}$ has absorbed the constants and taken care of the inversion.

The foregoing reasoning simply provides justification for testing relation 4; that is, that revolutions per foot is a function of $U \sqrt{\rho}$ only. The test is applied in figures 5 and 6 , where the results of figures 3 and 4 have been replotted with revolutions per foot as ordinates against $U \sqrt{\rho}$ as abscissas. It is seen that the curves for water and air have been brought into close agreement, approximating the condition of a single curve, as indicated by relation 4 . In figure 5 (also in fig. 3) the two curves for air show the marked effect of changing

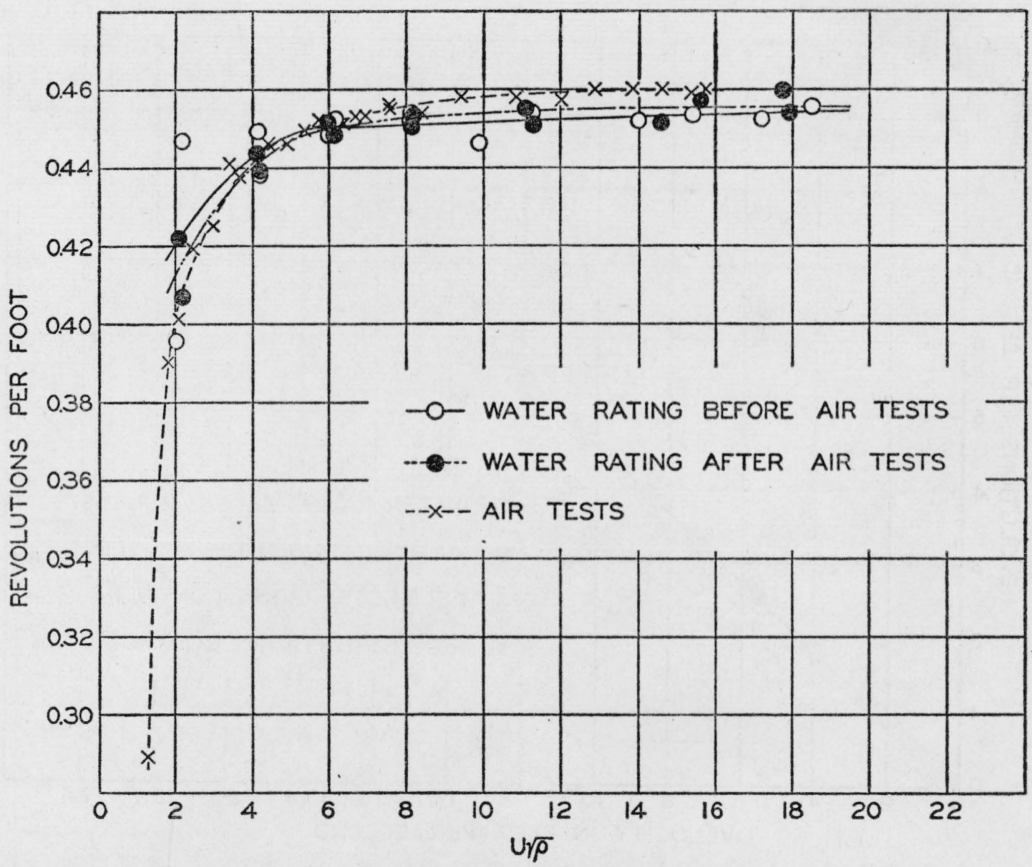

Figdre 6.-Relation between revolutions per foot and $U \sqrt{\rho}$.

$U=$ velocity of water or air in feet per second.

$\rho=$ Density of water or air in pounds per cubic foot.

Meter WR-1496.

the tension of the contact brush and thereby changing $T$. In view of this sensitivity to small changes in friction and the drastic nature of the changes from water to air, it is not surprising that the over-all agreement is not closer than 1 percent. In fact, the approximation to a single curve must be regarded as remarkably good, a condition which amply supports the validity of relation 4 or the more general relation 3.

\section{DISCUSSION}

There is, therefore, an effect of density, the magnitude of which increases with $T$. As $T$ approaches zero, $T / \rho U^{2} D^{3}$ approaches zero, and the revolutions per foot will show less and less dependence on both $\rho$ and $U$; that is, curves similar to those of figures $3,4,5$, and 6 will show less and less tendency to drop at low values of $U$ or $U \sqrt{\rho}$. 
If instead of decreasing $T / \rho U^{2} D^{3}$ by decreasing $T$, we do so by increasing $\rho$, the effect is the same. Of the latter we have examples in figures 3 and 4, where it may be seen that the flat, horizontal part of the curves persists to lower velocities for the case where the density is the higher. Further increases in density would simply push the knee of the curve to still smaller velocities. It is obvious at once that a density greater than that of water can have very little effect on the performance of the meters, except at very low velocities. For example, it may be seen by the aid of either figures 5 or 6 that if either of these meters were run in a fluid twice as dense as water flowing with a velocity of 0.5 feet per second, the rates would be only about

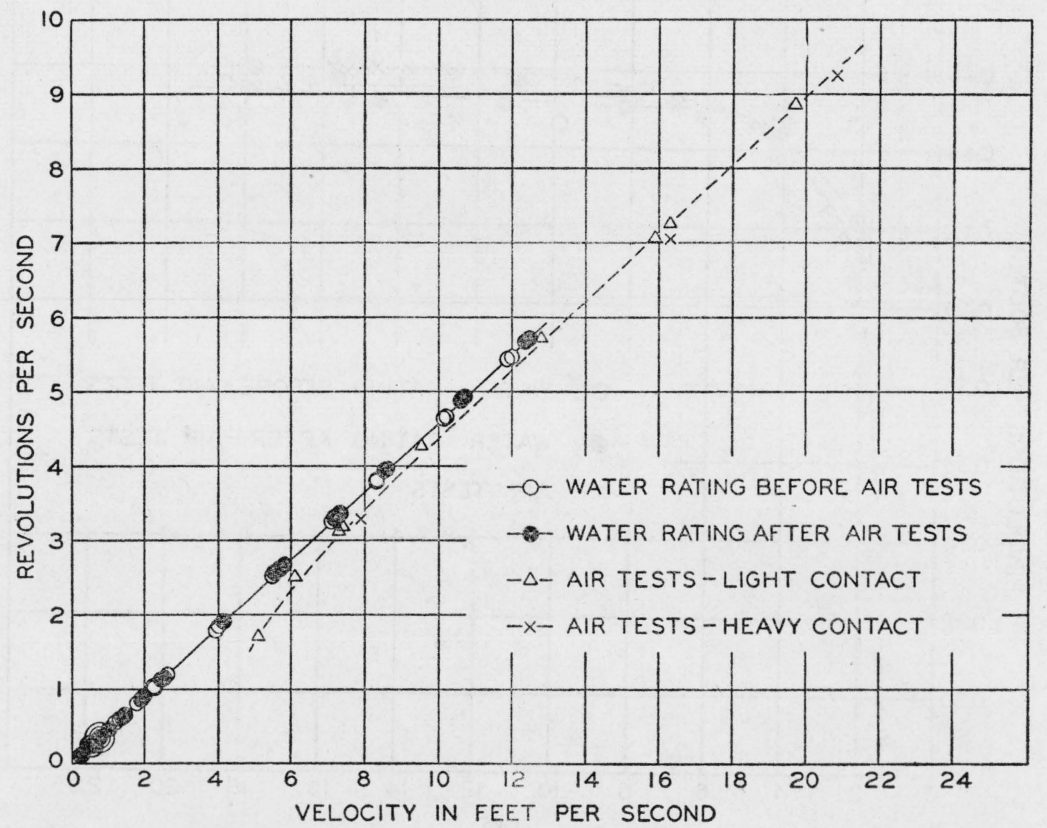

FIGURE 7.-Rating curves showing relation between revolutions per second and velocity of water and air.

Meter WR-1495.

2 percent higher than they would be in water. At higher velocities the difference would be still less.

Lowering the density will, in general, have a greater effect than increasing the density, but as long as the density is near that of water the effect will not be large. As a second example, suppose the density of the fluid to be half that of water. Again, using figures 5 or 6 , we find that the rate of a meter in such a fluid flowing with a velocity of 0.5 feet per second would be lowered by 3 percent below that for water. Since halving the density has the same effect as doubling the frictional torque, we may apply the example to a meter which for some reason has had its bearing friction doubled. Under the conditions of higher friction the rate of this meter would be lowered by 3 percent when running in water flowing at 0.5 feet per second. At higher velocities the change would be less. 
This condition is further illustrated by figures 7 and 8 , which show the usual type of rating curve. In these figures the curves giving the water rating do not depart perceptibly from a straight line, but actually if plotted to a more open scale the lines could be seen to curve toward the velocity axis at the low velocities, in the manner of the curves giving the air rating, and to intercept the abscissa at the starting velocity. A fluid with a greater density than that of water would yield a curve with a straight portion extending to still smaller velocities and having a smaller intercept on the velocity axis. Since the line is already quite straight it is apparent that further increase of density can have little effect. However, decreasing the density or

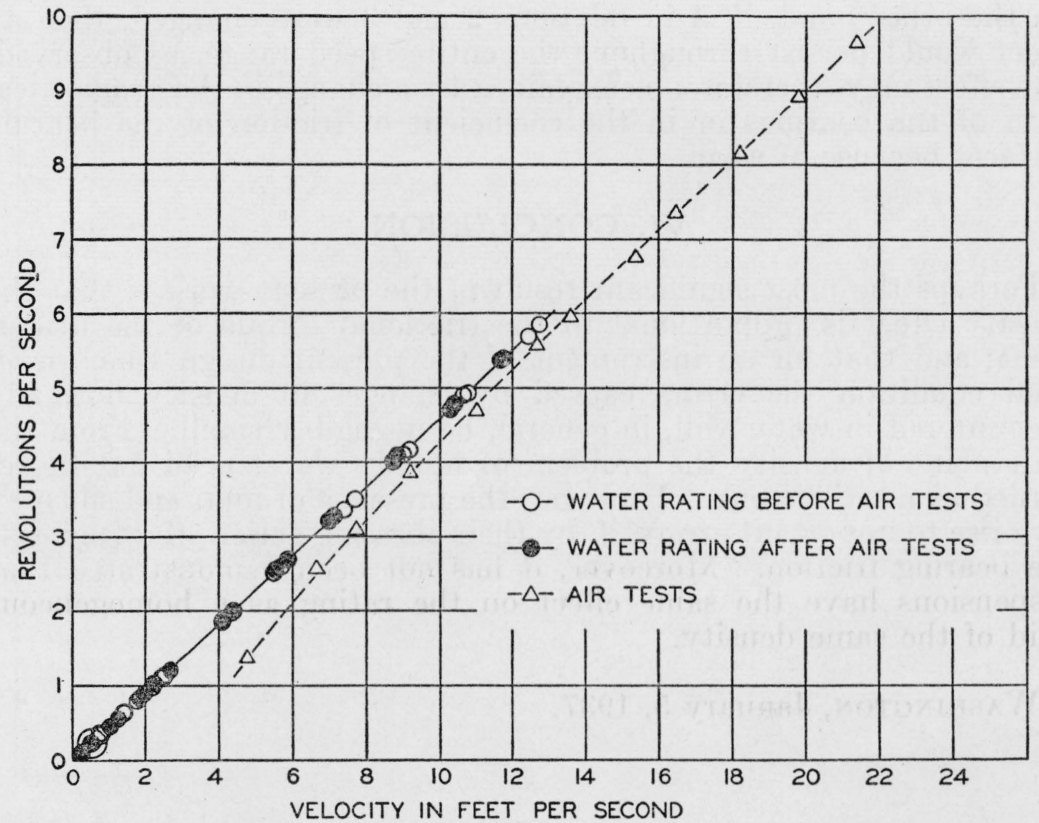

FIGURE 8.-Rating curves showing relation between revolutions per second and velocity of water and air.

Meter WR-1496.

increasing the friction can have an effect of any magnitude since the line can be given any amount of curvature.

If it is desired to obtain a rating curve, such as shown in either figures 7 or 8 , for some density other than that for which the meter was calibrated, the following procedure may be adopted. From calibration data consisting in rates of rotation of the cup wheel corresponding to known velocities and the known density of the fluid, a curve like those shown in either figures 5 or 6 is computed. Corresponding to any value of the ordinate a new velocity $U_{1}$ is computed by

$$
U_{1} \sqrt{\rho_{1}}=U_{c} \sqrt{\rho_{c}},
$$

where $U_{c}$ and $\rho_{c}$ are the velocity and density pertaining to calibration and $U_{1}$ is the velocity at the density $\rho_{1}$ which will yield the same value 
of the ordinate, i. e., the same value of revolutions per foot. Having $U_{1}$, the new rate of rotation corresponding to it is revolutions per second $=U_{1} \times$ revolutions per foot.

In this way a new set of rates and velocities corresponding to the density $\rho_{1}$ may be calculated and the new rating curve obtained.

An examination of figures 3 and 4 will reveal the fact that both meters ran faster throughout the velocity range during the second test in water following the tests in air. This phenomenon has been observed generally when meters have been recalibrated after much use. The effect is usually attributed to "running in", a condition of wear which favors faster running. If $T$ were decreased due to wear, the increased rate would be noticeable only at the lower velocities. On the other hand, if $A$ in relations 2 and 3 were changed, then an effect would persist throughout the entire speed range, as observed. The effect may, therefore, be explained by a change in the geometrical form of the bearings or in the coefficient of friction of the bearing surfaces because of wear.

\section{CONCLUSION}

Perhaps the most significant result of the present work is that the density effect is proportional to the frictional torque of the instrument; and that for an instrument of the present design which is in good condition the errors caused by changes in density normally encountered in water will, in general, be negligibly small. From the standpoint of density the problem of muddy water need not be regarded as a serious one. However, the presence of mud and silt may give rise to important errors, if, by their abrasive action, they increase the bearing friction. Moreover, it has not been demonstrated that suspensions have the same effect on the rating as a homogeneous fluid of the same density.

Washington, January 5, 1937. 